\title{
DETERMINATION OF THE SIZE OF EXPERIMENT
}

\author{
AKIRA SAKUMA* \\ Department of Pharmacology, University of Michigan Medical School, Ann Arbor, Michigan, U.S.A. \\ Received for publication July 21,1961
}

In the statistical evaluation of the superiority of one drug over another drug two types of problems arise. The first type of problem is that we may erroneously accept that a true difference exists between drug $\mathbf{A}$ and drug $B$ when in fact no true difference exists. The second type of problem is the failure to detect a difference when in fact the two drugs are truly different. The first type of error is the one most frequently reported in which the 'P' $(\alpha)$ value expresses the probability that a difference as large as the observed difference could have arisen fortuitously. This type of error is the one that concerns most investigators because it indicates the risk involved in rejecting that a true difference does not exist. The second type of error is of less importance to the average investigator. It is, however, of more obvious importance to investigators who are engaged in the detection of new drugs. Here the investigator wishes to know the chance of overlooking a potentially useful drug. He must weigh the expense of further investigation against the risk of overlooking a potentially useful drug.

It is evident that as the number of replications in a series of experiments is increased the more reliable will be the results; a potentially useful drug will be lesslikely discarded. The number of replications, however, is often limited by such factorsas expense, reproducibility of experimental conditions, length of experiments, etc. The expenses for animals and other materials may increase proportionally to the number of replications, whereas the unlikeliness of overlooking a useful drug is not a linear function of the number of experiment. Maintenance of stable conditions throughout a large series of experiments is rather difficult. It takes a long period of time to carry out a large number of experiments. If it is desired to obtain the results as quickly as possible, this time factor would be an important limiting step in designing the size of experiment. This may also interfere with maintenance of stable conditions.

Thus, it is generally desirable to finish a series of experiments with a minimum possible number of replications at a low level of the second type of error. Predetermination of an approximate minium size of experiment is possible when some information is available and some criteria are established. This type of study has been developed by statisticians as OC (operating characteristic) curves. The curves presented in this paper were calculated according to the formula described by Cochran and Cox (1). These curves will give an

\footnotetext{
* Fulbright Travel Grantee, 1958-60. Present address : Department of Pharmacology, Faculty of Medicine, University of Tokyo, Bunkyo-ku, Tokyo.
} 
approximation of the minimum number of replications required in experiments such as those-that can be statistically analyzed by routine $t$-tests or by some types of analyses of variance. Similar kind of information has been available for pharmacologists, e.g., on quantal responses (2) and parallel line assays (3).

In some cases where the technique of sequential analysis is feasible further reduction in the size of experiment may be expected, although the procedure is somewhat complicated and still under development (4-6).

\section{GENERAL REQUIREMENTS}

The following criteria are necessary to predetermine the number of replications: a) The experimental error is distributed approximately normally and homogeneously; otherwise an estimate of the number would be poor. Some special transformation of the data into metameters may be useful in obtaining a homogeneous, normal distribution of the error (7). b) An error of the first kind, $\alpha$, is assigned specifying either a one- or two-tailed test. The one-tailed test is used when the investigator is. interested in whether the treatment effect is larger than the control effect, or conversely whether the treatment effect is smaller than the control. The two-tailed test is used when the investigator is concerned only whether the treatment effect is different from the control effect without specifying larger or smaller. c) An error of the second kind, $\beta$, is designated. Upon the statistical test of the difference between treatment and control means, the null-hypothesis, Ho, is $\overline{\mathbf{X}}_{\mathrm{T}}=\overline{\mathbf{X}}_{\mathbf{C}}$ (two-tailed test). If one rejects the Ho and accepts the alternative hypothesis, $\mathrm{Ha}: \overline{\mathbf{X}}_{\mathrm{T}} \neq \overline{\mathbf{X}}_{\mathrm{C}}$, when the $\mathrm{H}_{\mathrm{o}}$ is true the risk involved in rejecting the true Ho is $\alpha$. Furthermore, if one does not reject the Ho when the $\mathrm{Ha}$ is true, the risk in accepting the false $\mathrm{Ho}$ is $\beta$. d) A ratio, $\lambda$, is established depending upon the worker's purpose. $\lambda$ is defined as a hypothetical difference between the control and treatment means divided by the standard deviation of the series of experiments. If one would like to detect a difference between two means as great as one standard deviation, the value of $\lambda$ is designated as 1 . In some cases where the estimation of the standard deviation is possible the minimum difference that one would like to detect can also be expressed as an actual value. For example, if one wanted to detect a difference of $15 \mathrm{mmHg}$ of vasoconstrictor effect between drugs $A$ and $B$ in a series of experiments whose standard deviation was estimated as $10 \mathrm{mmHg}$, the value of $\lambda$ is $15 / 10$ or 1.5 .

\section{EXAMPLES}

The approximate relationships among $\alpha(0.05), \beta, \lambda$, and $\mathrm{N}$, the number of replications, can be obtained from the curves: Figs. 1 and 2 illustrate the $O C$ curves when one- and two-tailed tests are employed, respectively. The ordinate $\beta$ expresses the probability of accepting Ho. The abscissa is indicated in terms of $\lambda$ which is defined as the hypothetical mean difference divided by the standard deviation.

a) Known versus Unknown. Drug. $\mathrm{A}$ is a well-known vasoconstrictor agent. This produces a $30 \mathrm{mmHg}$ rise in blood pressure in a given dose. The standard deviation of 

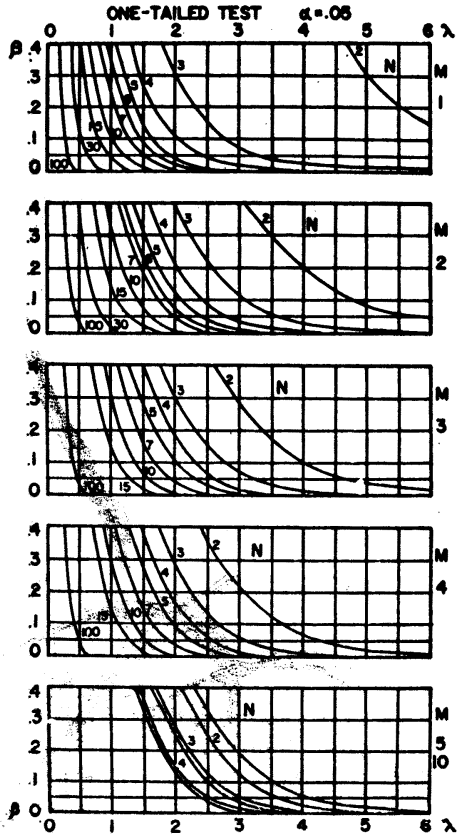

FIG. 1
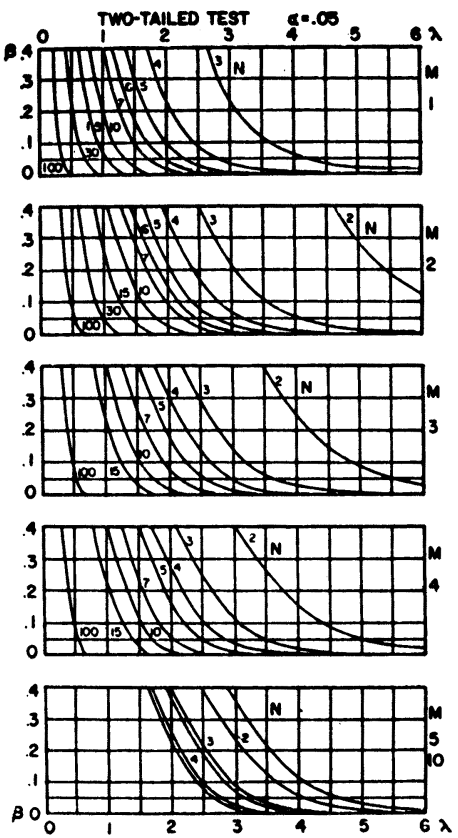

Fig. 2

Approximate relationships among $\alpha(0.05), \beta, \lambda$, and $\mathrm{N}$ can be obtained from Fig. 1 (one-tailed test) and Fig. 2 (two-tailed test). M1 is used for known vs. unknown ; M2 for two unknowns ; MR for one-way classification of $R$ items ; and $M(R-1)$ for two-way classification of $R$ items. In the chart $\mathrm{M} 5,10$, the right curve corresponds to $\mathrm{M} 5$ and the left to M10 in each $\mathrm{N}$.

this type of experiment has been estimated to be $5 \mathrm{mmHg}$. A new congener, drug $\mathrm{B}$, is to be tested. It is decided that studies on the new drug should be continued if an equimolar dose of the new drug is capable of producing a blood pressure rise of more than $35 \mathrm{mmHg}$. Thus, this practical criterion gives $\lambda=(35-30) / 5=1$. Entering the chart one-tailed, Ml (Fig. 1), one will find that the number of replications is 15 at $\beta=0.05$. These values, for example, suggest that i) if the one-tailed test of the results of 15 experiments with drug $B$ turns out significant at $\alpha=0.05$ (using $t$ at $\alpha=0.1$ in an ordinary two-tailed table, since the test is one-tailed), rejection of the Ho: drug B is no more potent than drug $\mathrm{A}$, involves a risk of $5 \%(\alpha)$, and that ii) if drug $\mathrm{B}$ actually produces a blood pressure rise of more than $35 \mathrm{mmHg}$ in the long run, the chance that it will not be found statistically superior to drug $\mathrm{A}$ is $5 \%(\beta)$. In other words, the experimenter is unlikely to discard a new drug $B$ if it is really superior to drug $A$ by $5 \mathrm{mmHg}$.

b) Two Unknowns (Group Comparison). Drugs A and B are bactericidal agents. Antibacterial effectiveness is expressed as the decrease in the number of colonies. A pilot study suggested that the standard deviation of this type of experiment would be about 15. How many replications are necessary to detect the difference of at least 5 colonies between two agents? Form the chart two-tailed, M2 (Fig. 2), the number of each group of experiments would be determined to be more than 100 at $\alpha=0.05, \beta=0.1$, and $\lambda=5 / 15$. Thus, this technique would be practically useless as a quick, inexpensive screening to 
differentiate drugs under the abovementioned conditions. The following alternatives are possible: i) To find out some experimental technique with smaller standard deviation (improvement in technique); and ii) To change the minimum difference that one would like to detect, or to change the levels of $\alpha$ and $\beta$ (alterations in criteria).

c) One-Way Classification. This is an extended form of the group comparison. Four hypotensive drugs, in general $\mathbf{R}$ drugs, are to be compared in a one-way classification analysis. The results would be arranged in a table and sums of squares are derived as follows :

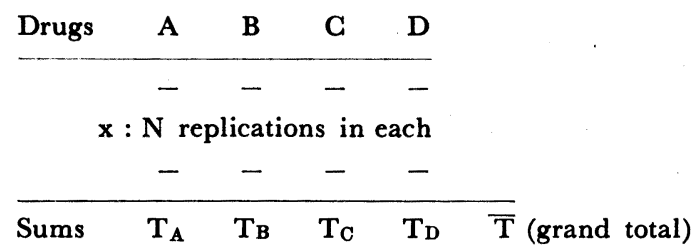

CF (correction factor) $=\overline{\mathrm{T}}^{2} /(\mathrm{RN})$

$\mathrm{S}_{\text {total }}=\sum \mathrm{x}^{2}-\mathrm{CF}$
$\mathrm{S}_{\text {drug }}=1 / \mathrm{N} \sum \mathrm{T}^{2}-\mathrm{CF}$
$\mathrm{S}_{\text {residual }}=\mathrm{S}_{\text {total }}-\mathrm{S}_{\text {drug }}$

An unbiased estimate of standard deviation is

$$
\mathrm{s}=\left[\mathrm{S}_{\text {residual }} / \mathrm{R}(\mathrm{N}-1)\right]^{1 / 2}
$$

The significance test for difference between any two means is carried out by the least significant difference method (8), that is, the test for the difference between the means of drugs $I$ and $J$ is significant if the squared difference between the two sums, $\left(T_{I}-T_{J}\right)^{2}$, exceeds a critical value, $D^{2}$, defined as

$$
\mathrm{D}^{2}=2 \mathrm{~N} t^{2} \mathrm{~s}^{2}
$$

where $t$ is a Student's $t$ at $\alpha$, with the degrees of freedom, Df, of $\mathrm{R}(\mathrm{N}-1)$. One may make use of a relationship that

$$
t^{2}(\mathrm{Df})=F(1, \mathrm{Df})
$$

The chart two-tailed, M4 (Fig. 2), shows that the number of replications in each drug should be at least 6 or 7 to detect a difference of two standard deviations between any two drug effects at $\alpha=0.05$ and $\beta=0.1$. In the one-way classification analysis the chart (one- or two-tailed) MR should be used when $\mathrm{R}$ items are involved. In the cases of larger R's one may use the chart M4, since the OC curves are almost the same of larger R's, particularly if $\mathrm{N}$ is larger than 4 or 5 . M5, 10 shows these relationships.

d) Two-Way Classification. A two-way classification analysis is an extension of the

\begin{tabular}{lc|cccc|c} 
Drugs & & $\mathbf{A}$ & $\mathbf{B}$ & $\mathbf{C}$ & $\mathbf{D}$ & Sums \\
\hline Animals & $\mathbf{1}$ & - & - & - & - & $\mathbf{t}_{1}$ \\
& 2 & - & - & - & - & $\mathbf{t}_{2}$ \\
& $:$ & $:$ & $\mathbf{x}$ & $:$ & $:$ & $:$ \\
& $\mathbf{N}$ & - & - & - & - & $\mathbf{t}_{\mathbf{N}}$ \\
\hline \multicolumn{2}{c|}{ Sums } & $\mathbf{T}_{\mathbf{A}}$ & $\mathbf{T}_{\mathbf{B}}$ & $\mathbf{T}_{\mathbf{C}}$ & $\mathbf{T}_{\mathbf{D}}$ & $\overline{\mathbf{T}}$ (grand total)
\end{tabular}


paired comparison in which only two items are compared. Again four drugs, in general $\mathrm{R}$ drugs, are to be tested in a twc-way layout. This type of design differs from the one-way classification being cross-classified as shown in the table.

Sums of squares are derived as follows :

$$
\begin{aligned}
& \mathrm{CF}=\overline{\mathrm{T}}^{2} /(\mathrm{RN}) \\
& \mathrm{S}_{\text {total }}=\sum \mathrm{x}^{2}-\mathrm{CF} \\
& \mathrm{S}_{\text {drug }}=1 / \mathrm{N} \sum \mathrm{T}^{2}-\mathrm{CF} \\
& \mathrm{S}_{\text {animal }}=1 / \mathrm{R} \sum \mathrm{t}^{2}-\mathrm{CF} \\
& \mathrm{S}_{\text {residual }}=\mathrm{S}_{\text {total }}-\mathrm{S}_{\text {drug }}-\mathrm{S}_{\text {animal }}
\end{aligned}
$$

An unbiased estimate of standard deviation is

$$
\mathrm{s}=\left[\mathrm{S}_{\text {residual }} /(\mathrm{R}-1)(\mathrm{N}-1)\right]^{1 / 2}
$$

A critical value for the test of the least significant difference is

$$
\mathrm{D}^{2}=2 \mathrm{~N} t^{2} \mathrm{~s}^{2}
$$

where $t$ is now with $\mathrm{DF}$ of $(\mathrm{R}-1)(\mathrm{N}-1)$. For example, the difierence, $\left|\mathrm{T}_{\mathrm{A}}-\mathrm{T}_{\mathrm{B}}\right|$, is compared with $|\mathrm{D}|$ to examine whether the mean effects of drugs $\mathrm{A}$ and $\mathrm{B}$ are singnificantly different.

In the two-way classification analysis with $R$ items, the chart $M(R-1)$ should be used. Thus, the chart one- or two-tailed, M3 is used for the abovementioned example. The paired comparison in which the number of items is two is the simplest from of the twoway classification, and one may use chart M1 to design the experiment.

\section{SUMMARY}

The use of OC curves was illustrated with special reference to designing the size of experiment whose results can be statistically analyzed by $t$-tests or by analyses of variance. These curves provide the level of the error of the second kind, $\beta$, when the statistical test of the results turns out non-significant.

Acknowledgment. The author wishes to thank Dr. William G. Cochran for his helpful suggestions about type II error.

\section{REFERENCES}

1) Gochran, W.G. And Gox, G.M. : Experimental Designs, Chapt. 2.2, John Wiley \& Sons, New York (1957)

2) Kramer, M. AND Greenhouse, S.W. : Determination of Sample Size and Selection of Cases, in Psychopharmacology Problems in Evaluation, National Academy of Sciences-National Research Council (1959)

3) Sheps, M.C. And Hendrie, K.H. : J. Pharmacol. 124, 94 (1958)

4) Gochran, W.G. : Newer Statistical Methods, in Quantitative. Methods in Human Pharmacology and Therapeutics, Pergamon Press, London (1959)

5) Cummings, J.R., Haynes, J.D., Lipahuak, L.M. and Ronsberg, M.A. : J. Pharmacol. 128, 414 (1960)

6) Lasagna, L. And Meier, P. : Experimental Design and Statistical Problems, in Clinical Evaluation of New Drugs, Hoeber-Harper Book, New York (1959)

7) Gaddum, J.H. : Pharmacol. Rev. 5, 87 (1953)

8) DuncaN, A.J. : Quality Control and Industrial Statistics, Richard, D. Irwin, Homewood, 111. (1959) 